\title{
Cryo-EM structure of an early precursor of large ribosomal subunit reveals a half-assembled intermediate
}

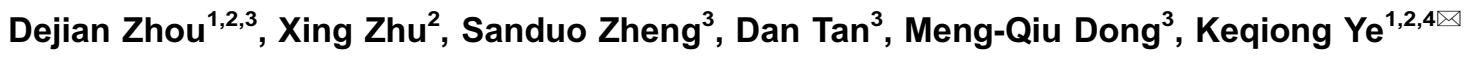 \\ ${ }^{1}$ Graduate School of Peking Union Medical College and Chinese Academy of Medical Sciences, Beijing 100730, China \\ 2 Key Laboratory of RNA Biology, Institute of Biophysics, CAS Center for Excellence in Biomacromolecules, Chinese Academy \\ of Sciences, Beijing 100101, China \\ ${ }^{3}$ National Institute of Biological Sciences, Beijing 102206, China \\ ${ }^{4}$ University of Chinese Academy of Sciences, Beijing 100049, China \\ $\triangle$ Correspondence: yekeqiong@ibp.ac.cn (K. Ye)
}

Received January 9, 2018 Accepted March 4, 2018

\begin{abstract}
Assembly of eukaryotic ribosome is a complicated and dynamic process that involves a series of intermediates. It is unknown how the highly intertwined structure of 60 S large ribosomal subunits is established. Here, we report the structure of an early nucleolar pre-60S ribosome determined by cryo-electron microscopy at $3.7 \AA$ resolution, revealing a half-assembled subunit. Domains I, II and VI of 25S/5.8S rRNA pack tightly into a native-like substructure, but domains III, IV and V are not assembled. The structure contains 12 assembly factors and 19 ribosomal proteins, many of which are required for early processing of large subunit rRNA. The Brx1-Ebp2 complex would interfere with the assembly of domains IV and V. Rpf1, Mak16, Nsa1 and Rrp1 form a cluster that consolidates the joining of domains I and II. Our structure reveals a key intermediate on the path to establishing the global architecture of 605 subunits.
\end{abstract}

KEYWORDS ribosome assembly, cryo-EM, pre-60S ribosome, nucleolar

Dejian Zhou and Xing Zhu have contributed equally to this work.

Electronic supplementary material The online version of this article (https://doi.org/10.1007/s13238-018-0526-7) contains supplementary material, which is available to authorized users.

\section{INTRODUCTION}

Correct formation of the ribosome is essential for protein synthesis in cells. The ribosome is composed of a small $40 \mathrm{~S}$ subunit and a large 60 S subunit (SSU and LSU) in eukaryotes. Ribosome assembly is a highly complicated process that engages more than 200 protein assembly factors (AFs) and many snoRNAs (Woolford and Baserga, 2013). These factors temporarily associate with ribosomal subunits and drive their maturation. In the yeast $S$. cerevisiae, assembly of both subunits begins in the nucleolus with the transcription of a long precursor rRNA (pre-rRNA) that encodes 18S, 5.8S and $25 \mathrm{~S}$ rRNAs and four external and internal transcribed spaces (ETS and ITS). The $5^{\prime}$ region of the pre-rRNA is cotranscriptionally assembled into the $90 \mathrm{~S}$ pre-ribosome or SSU processome, which is the early precursor of SSU (Dragon et al., 2002; Grandi et al., 2002; Osheim et al., 2004; Chaker-Margot et al., 2015; Zhang et al., 2016). Following cleavage of the pre-rRNA at the A0, A1 and A2 sites, the $90 \mathrm{~S}$ pre-ribosome is released from the pre-rRNA and transformed into a pre-40S ribosome. Cryo-EM structures of $90 \mathrm{~S}$ pre-ribosome show that the nascent $40 \mathrm{~S}$ subunit is assembled into several isolated units that have yet to acquire the global architecture of $40 \mathrm{~S}$ subunit (Kornprobst et al., 2016; Barandun et al., 2017; Chaker-Margot et al., 2017; Cheng et al., 2017; Sun et al., 2017).

The $60 \mathrm{~S}$ subunit is composed of $25 \mathrm{~S}, 5.8 \mathrm{~S}$ and $5 \mathrm{~S}$ rRNAs, and 46 ribosomal proteins (RPLs). The 25S/5.8S rRNAs are divided into six domains that intertwine to form a monolithic structure (Ben-Shem et al., 2011; Klinge et al., 2011). The earliest pre-60S ribosome is co-transcriptionally 
formed on the $3^{\prime}$ LSU region of pre-rRNA in a stepwise manner (Chen et al., 2017). The pre-60S progressively matures as it transits from the nucleolus to the nucleoplasm and cytoplasm. The maturation of pre-60S is coupled to the dynamic association of approximately 90 assembly factors, hierarchical incorporation of RPLs and sequential processing of ITS1 and ITS2. The development of pre-60S at the nucleoplasm and cytoplasm has been characterized to great extents, particularly by recent cryo-EM studies (Pena et al., 2017). An early nucleoplasmic state is characterized by a foot structure assembled on the partially processed ITS2 and an immature central protuberance (CP) (Bradatsch et al., 2012; Leidig et al., 2014; Wu et al., 2016). Further evolvement of pre-60s leads to removal of the foot, maturation of the CP and association of the Rix1-Ipi1-Ipi2 subcomplex and the AAA-ATPase Rea1 (Barrio-Garcia et al., 2016). Rea1 hydrolyzes ATP and drives the release of Ytm1 and Rsa4 (Ulbrich et al., 2009; Bassler et al., 2010). The pre-60S acquires competence for nuclear export at the late nucleoplasmic stage and undergoes final maturation in the cytoplasm (Matsuo et al., 2014; Greber et al., 2015; Weis et al., 2015; Ma et al., 2017; Malyutin et al., 2017).

In all previously determined cryo-EM structures of pre605 , the structural core has already assumed the shape of mature subunit. Therefore, the key question of how the global architecture of LSU is established is still unknown. In this study, we determine the cryo-EM structure of pre-60S at an early nucleolar state. The structure is only half-assembled at domains I, II and VI and represents a key intermediate in the formation of global architecture of LSU.

\section{RESULTS}

\section{Structure determination}

We affinity purified pre-60S using the bait protein Rpf1 fused to a C-terminal tandem affinity purification (TAP) tag. Mass spectrometry analysis showed that the Rpf1-TAP particle displayed a similar protein composition as the previously characterized nucleolar Ssf1-TAP and Nsa1-TAP particles (Fatica et al., 2002; Kressler et al., 2008; Chen et al., 2017) (Fig. S1, Supplementary Dataset 1). Cryo-EM images were collected in a Titan Krios $300 \mathrm{kV}$ electron microscope equipped with a direct electron detector (Fig. S2). After three rounds of 3D classification, 98,155 particles were selected to reconstruct a density map at an overall resolution of $3.65 \AA$ (Figs. 1A and S3). Large side chains of protein and RNA bases are resolved in the structure core, whereas some peripheral regions including many AFs are of lower resolution (Figs. S3 and S4). The structure was built based on the cryo-EM structure of Nog1-TAP pre-60S (Wu et al., 2016), crystal structures (Brx1, Ebp2, Nsa1), homology models (Has1 and Rpf1) and de novo modeling (Mak16 and Rrp1) (Fig. S4 and Table S1). The Rpf1-TAP sample was also analyzed by chemical crosslinking and mass spectrometry (CXMS), yielding 28 intermolecular and 110 intramolecular crosslinks (Supplementary Dataset 2, Fig. S5). These intermolecular crosslinks assisted the assignment of AFs (Table S1) and also suggested the position of unmodeled AFs, such as Dbp10, Drs1, Nop2 and Mak11 (Fig. S5). The final model was refined in real space and displayed good geometry (Table S2).

\section{Overall structure}

The cryo-EM density map and structural model of Rpf1-TAP pre-60S are shown in Fig. 1. The most striking feature of the structure is that only half of LSU is present. Domains I, II and $\mathrm{VI}$ assemble into a native-like substructure, but domains III, IV and $\mathrm{V}$ are totally absent (Fig. 2). A few peripheral elements in domain II that form the CP (H38, ES12) and the P stalk $(\mathrm{H} 43, \mathrm{H} 44)$ and interact with domains III, VI and V (H33$\mathrm{H} 35)$ are also disordered (Fig. 2E). Therefore, the structure represents an early assembly intermediate before the global architecture of $60 \mathrm{~S}$ is established. A total of $12 \mathrm{AFs}$ and 19 RPLs were modeled in the map (Figs. 1B, 2C and 2D). Five AFs, namely, Nop7, RIp7, Nop15, Cic1 and Tif6, are also present in the early nucleoplasmic pre-60S (Bradatsch et al., 2012; Leidig et al., 2014; Wu et al., 2016) and adopt similar conformation in the Rpf1-TAP pre-60S. Seven AFs Has1, Brx1, Ebp2, Rrp1, Mak16, Nsa1 and Rpf1 were newly located (Fig. S3).

\section{Assembly of ITS2}

The N-terminal region of ITS2 is associated with Cic1, Nop15, RIp7 and Nop7, forming the foot structure (Figs. 1 and $3 A$ ). The foot structure is similar to that of the nucleoplasmic pre-60S structure (Bradatsch et al., 2012; Leidig et al., 2014; Wu et al., 2016), except that Nop53 is not present (Fig. 3B). Nop53 recruits the exosome for processing 7S pre-rRNA (Thoms et al., 2015; Falk et al., 2017). Nop53 is of low abundance in the Rpf1-TAP particle (Fig. S1) and likely associates at a later stage.

\section{Has1}

Has1 is a DEAD-box RNA helicase involved in both small and large ribosomal subunit assembly (Emery et al., 2004; Dembowski et al., 2013). RNA helicases are thought to unwind RNA structures or disassemble RNA-protein complexes, hence driving the evolvement of pre-ribosomes. In the cryo-EM structure, Has1 is situated next to Cic1 and interacts with $\mathrm{H} 16$ of $25 \mathrm{~S}$ rRNA through its two RecA domains (Fig. 3A). DEAD-box helicases unwind short RNA duplexes by local strand separation (Yang et al., 2007). In a proposed mechanistic model (Mallam et al., 2012), RecA2 first associates with the RNA duplex, and binding of ATP to RecA1 then induces closure of two RecA domains, causing RecA1 to displace one RNA strand. In the pre-60S structure, $\mathrm{H} 16$ is bound to Has 1 at a separate site from where singleand double-stranded RNAs are bound to DEAD-box 

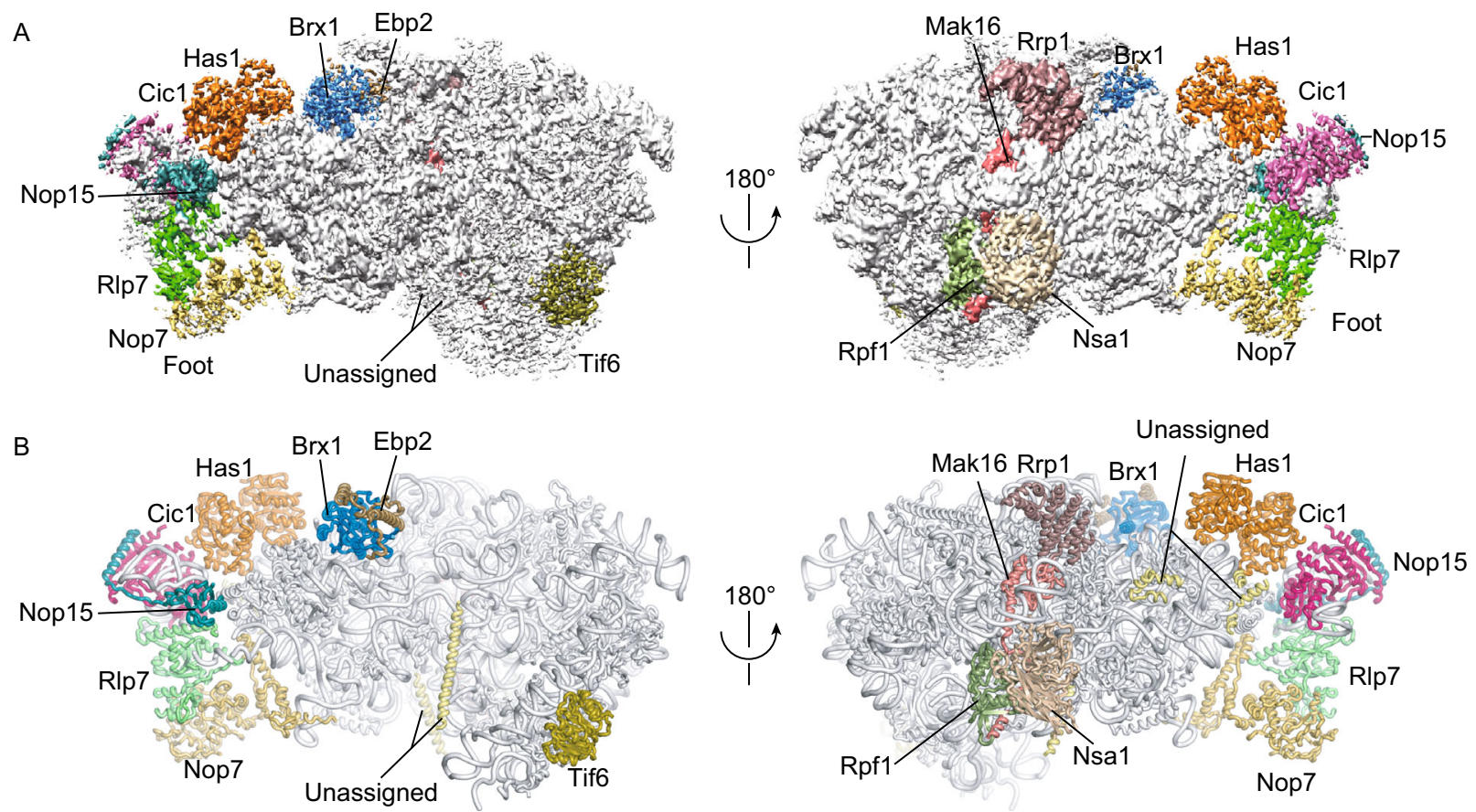

Figure 1. Cryo-EM structure of Rpf1-TAP pre-60S. (A) Cryo-EM density map in two opposite views. The densities for AFs are color coded. (B) Structural model in the same views as (A). The rRNAs and RPLs are colored grey and the AFs are color coded.

helicases (Fig. 3C and 3D) (Del Campo and Lambowitz, 2009; Mallam et al., 2012). In addition, the ATP-binding pocket of Has 1 is empty, and its two RecA domains are not arranged in the same closed conformation as in ternary complex structures of helicase, ssRNA and ATP analog. These structural features suggest that $\mathrm{H} 16$ serves as a docking site rather than an unwinding substrate for Has 1.

\section{Structure of the Brx1 and Ebp2 complex}

$\mathrm{Brx} 1$ is a Brix domain protein that interacts with Ebp2 (Wehner and Baserga, 2002; Shimoji et al., 2012). We determined the crystal structure of Brx1 (residues 26-255) in complex with Ebp2 (residues 195-293) at $2.3 \AA$ resolution with de novo phasing (Table S3). Brx 1 adopts an $\alpha / \beta$ fold with pseudo 2-fold symmetry (Fig. 4A), similar to other Brix domain proteins ( $\mathrm{Ng}$ et al., 2005; Asano et al., 2015; Kharde et al., 2015; Madru et al., 2015; Sa-Moura et al., 2017). The structural core of $B r x 1$ is composed of two curved $\beta$-sheets that form a half-open $\beta$-barrel sandwiching two $\alpha$-helices ( $\alpha 1$ and $\alpha 3$ ). The surface of the $\mathrm{N}$-terminal sheet holds helix $\alpha 2$, whereas the surface of the C-terminal sheet makes an intermolecular interaction with helix $\alpha 2^{\prime}$ of Ebp2. In addition, the Ebp2 sequences that flank helix $\alpha 2^{\prime}$ wrap around the Brx1 structure, forming an extensive interface. The $\alpha 2^{\prime}$ helix of Ebp1 packs against the N-terminal sheet of $\mathrm{Brx} 1$, and the $\beta 1$ ' strand of Ebp2 expands the C-terminal sheet of Brx1.

In the cryo-EM map, the Brx1-Ebp2 complex perfectly fits into a density bound to domain I (Figs. 1 and S4B). Brx1 interacts with a flat surface formed by $\mathrm{H} 13$ and $\mathrm{H} 21$. The position of Brx1 would clash with $\mathrm{H} 68$ and $\mathrm{H} 88$ in domains IV and $V$ in the fully assembled $60 \mathrm{~S}$ (Fig. 3B). Thus, the Brx1Ebp2 complex must be released from the current position for ribosome assembly to proceed.

There are six Brix domain proteins, namely, Imp4, Rpf2, Rpf1, Brx1, Ssf1 and Ssf2, in the yeast S. cerevisiae. Imp4 is involved in small subunit assembly, whereas the others function in large subunit assembly. The structures of Rpf2, Imp4, Rpf1 (see below) and Brx1 have now been determined in the context of pre-ribosomes (Leidig et al., 2014; Kornprobst et al., 2016; Wu et al., 2016; Barandun et al., 2017; Chaker-Margot et al., 2017; Cheng et al., 2017; Sun et al., 2017), allowing for comparison of their conserved and variable features in protein and RNA recognition (Fig. 4B-E). In all four proteins, an $\alpha$-helix of their partner proteins binds with the C-terminal sheet of the Brix domain. Outside the core interface, the intermolecular interaction is highly variable. Brx1 and Rpf2 form an extensive interface with their binding partners Ebp2 and Rsr1, but Rpf1 and Imp4 primarily bind only a single $\alpha$-helix of Mak16 and Mpp10. The RNA binding interface is also extremely diverse for the four Brix proteins. No consensus sequence or structure is apparent for the bound RNAs. Nevertheless, one protein region that consistently binds RNA is located at the junction between strand $\beta 1$ and helix $\alpha 1$ and the turn connecting strands $\beta 3$ and $\beta 4$. The diverse interactions with protein and RNA allow each Brix protein to play specific roles in ribosome assembly. 


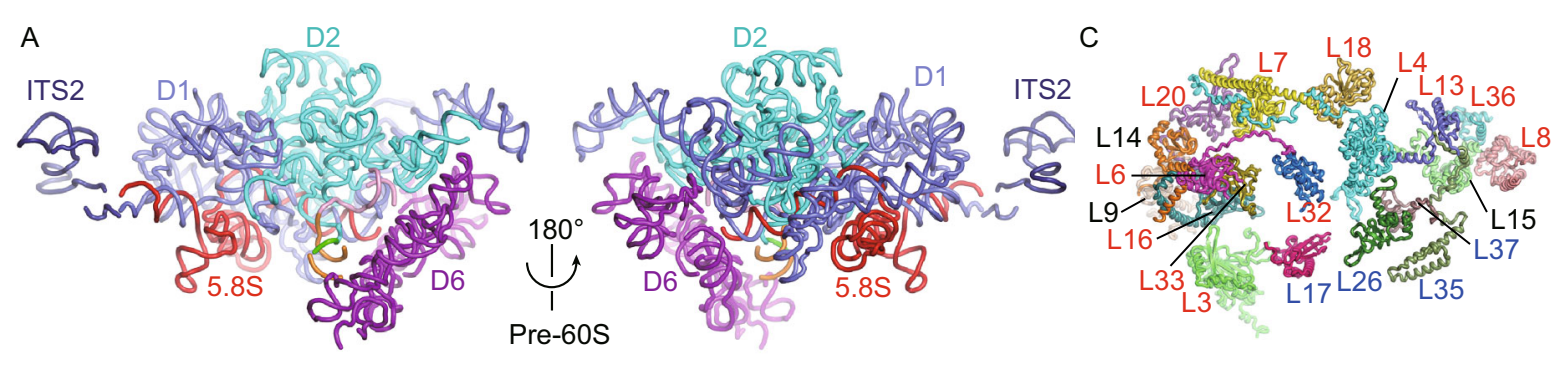

B
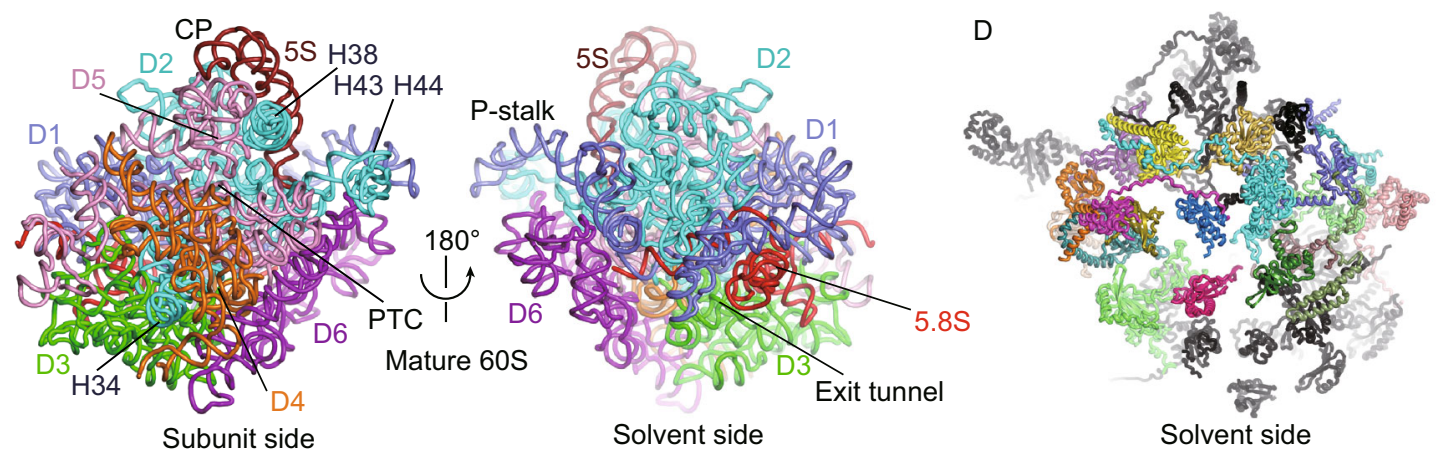

E

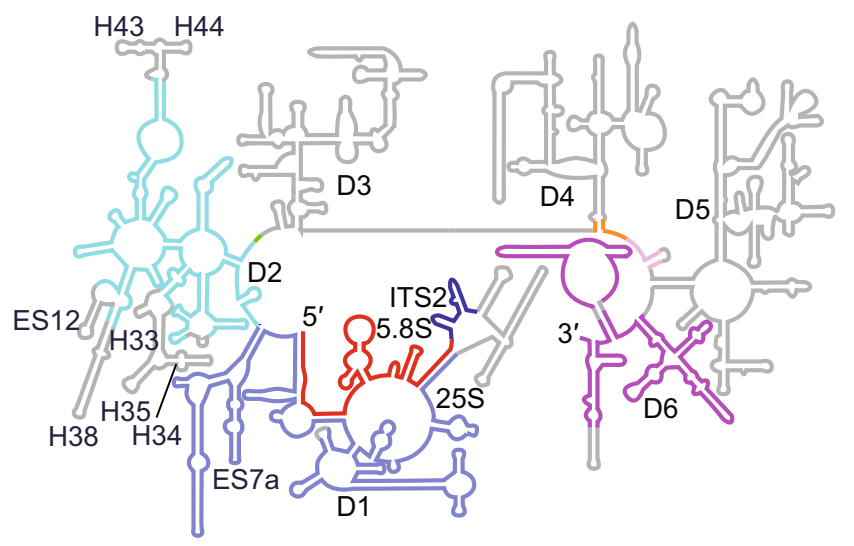

Figure 2. Structure of rRNAs and RPLs in Rpf1-TAP pre-60S and mature 60 S subunit. (A and B) Structure of rRNAs in Rpf1-TAP pre-60S (A) and mature 60S (B). The subunit and solvent side views are shown. Domains I-VI of $25 S$ are colored slate, cyan, green, orange, pink and purple, respectively. ITS2, 5.8S and 5S are colored dark blue, red and brown, respectively. Central protuberance (CP), peptidyl transferase center (PTC), polypeptide exit tunnel and P-stalk are labeled. (C and D) RPLs in Rpf1-TAP pre-60S (C) and mature 60S (D). The solvent side view is shown. RPLs present in pre-60S are color coded and those missing in pre-60S are shown in black in (D). The early, middle and unclassified RPLs are labelled in red, blue and black, respectively. (E) Secondary structure model of 5.8S, ITS2 and 25S RNA. The modeled regions are colored as in (A) and (B) and the unmodeled regions are colored gray.

\section{D2 factors}

Rrp1, Mak16, Rpf1 and Nsa1 form a cluster bound to a junction region of domains $\mathrm{I}$ and $\mathrm{II}$ at the solvent side (Fig. 5A-F). The four proteins are co-assembled into a prerRNA fragment ending at domain II (termed D2 factors) (Chen et al., 2017), consistent with their binding sites. These AFs are not present in the nucleoplasmic pre-60S and Nsa1 is released by the AAA ATPase Rix7 (Kressler et al., 2008).

Mak16 occupies a central position of the cluster and is mostly buried. Mak16 is composed of a knot-like N-terminal domain (NTD), a two-layered middle domain (MD) and an extended C-terminal helix (Fig. 5B). The NTD fits snugly into a cavity formed by $\mathrm{H} 45$ and $\mathrm{H} 46$ and ribosomal proteins $\mathrm{L} 4$ and L32. The MD is covered by the first helix of expansion segment 7 (ES7a) RNA. The C-terminal a-helix of Mak16 binds to the Brix domain protein Rpf1. This interaction was also demonstrated by yeast 2-hybrid, in vitro pull-down and gel filtration assays (Fig. S6) (McCann et al., 2015; Bassler et al., 2017). In addition to binding Mak16, Rpf1 also binds L32, Nsa1 and the base of helix ES7a. Nsa1 contains a WD domain (Lo et al., 2017) and is docked at H19, ES7a and Rpf1. Rrp1 folds into a super-helical structure and contacts L4, L18, the N-terminus of L7, Mak16 and RNA helix ES7a. By binding both domains I and II, these D2 factors appear to 

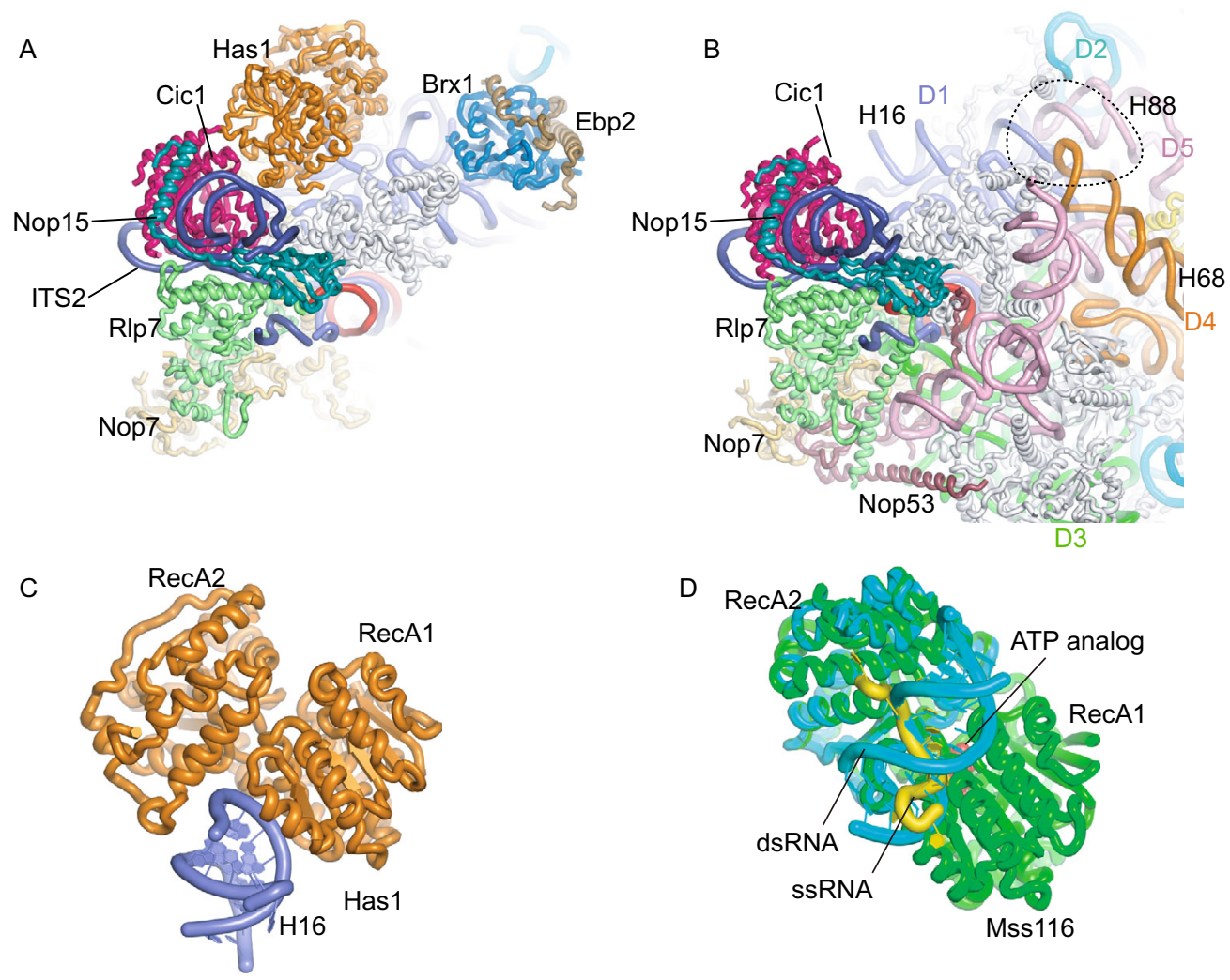

Figure 3. AFs bound to ITS2 and domain I. (A) Structure of Rpf1-TAP pre-60S near the ITS2. AFs, ITS2, 5.8S and individual domains of 25S rRNAs are color coded. All RPLs are shown in gray. (B) Structure of the Nog1-TAP pre-60S (PDB code: 3JCT) shown in the same view as (A). The dashed circle indicates the binding site of Brx1 and Ebp2 that would clash with $\mathrm{H} 68$ and $\mathrm{H} 88$. (C) Structure of Has1 bound to H16. (D) Superimposed structures of Mss116 in complex with ssRNA and dsRNA. Mss116 is aligned to Has1 in (C) by the RecA1 domain. The ternary complex structure (PDB code: 3I5X) is composed of Mss116 (green), ssRNA (yellow) and ATP analog (red). The binary complex containing the RecA2 domain of Mss116 bound to an RNA duplex (PDB code: 4DB2) is colored cyan and aligned to the ternary complex.

strengthen the joining of the two domains at early assembly stages when many interactions in the mature subunit are missing.

The ribosomal structure at the D2 factor binding region closely resembles the mature subunit (Fig. 5F). However, in the mature subunit, the $\mathrm{N}$-terminal tail of $\mathrm{L} 6$ inserts into the cavity initially occupied by Mak16, and ES7a becomes disordered in the absence of AF contacts. ES7a is a eukaryotespecific element in 25S rRNA and appears to function in ribosome assembly, which differs dramatically between bacteria and eukaryotes, rather than in protein translation.

\section{DISCUSSION}

We have determined the cryo-EM structure of pre-60S at an early nucleolar state. The structure demonstrates that the global architecture of $60 \mathrm{~S}$ is first established by domains I, II and $\mathrm{VI}$, which constitute the major part of the solvent shell of 60S. Part of the solvent shell (domain III) and the entire inner layer at the subunit interface (domains IV and V) have not been integrated. The functional sites of 605 , the peptidyl transferase center and the polypeptide exit tunnel, are not assembled at this stage. The absence of domains III, IV and $\checkmark$ does not mean that these domains are completely unfolded. They could fold and associate with AFs and RPLs (Chen et al., 2017) but are too flexible to be visualized in the cryo-EM structure. The Rpf1-TAP particle contains $\sim 40$ AFs of appreciable abundance (Fig. S1). Those AFs not present in our structure may be flexible or associate with other states of pre-60S. The map also contains unassigned structures and unmodeled weak densities.

In the Rpf1-TAP pre-60S, domains I, II and VI together with 19 RPLs are tightly packed as in the mature subunit. All AFs barely interfere on the formed ribosomal structure. By contrast, in the $90 \mathrm{~S}$ pre-ribosome, all four domains of the $40 S$ subunit are isolated and are organized by extensive interactions with a large number of AFs, the $5^{\prime}$ ETS and U3 snoRNA (Kornprobst et al., 2016; Barandun et al., 2017; Chaker-Margot et al., 2017; Cheng et al., 2017; Sun et al., 2017). 
Figure 4. Structure of the Brx1-Ebp2 complex.

(A) Crystal structure of the Brx1-Ebp2 complex shown in two opposite views. The $\mathrm{N}$ - and $\mathrm{C}$-termini and secondary structures are labeled. (B-D) Comparison of protein and RNA interactions of four Brix domain proteins in the context of pre-ribosome structures. Proteins are color coded and RNAs are colored in silver. The protein atoms within $5 \AA$ of any RNA atom are colored black. The Brx1-Ebp2 complex in the Rpf1-TAP pre-60S (B). The Rpf2Rrs1 complex in the Nog1-TAP pre-60S (PDB code: 3JCT) (C). The Rpf1-Mak16 complex in the Rpf1-TAP pre-60S (D). The Imp4-Mpp10 complex in the $90 \mathrm{~S}$ pre-ribosome (PDB code: 5WLC) (E).

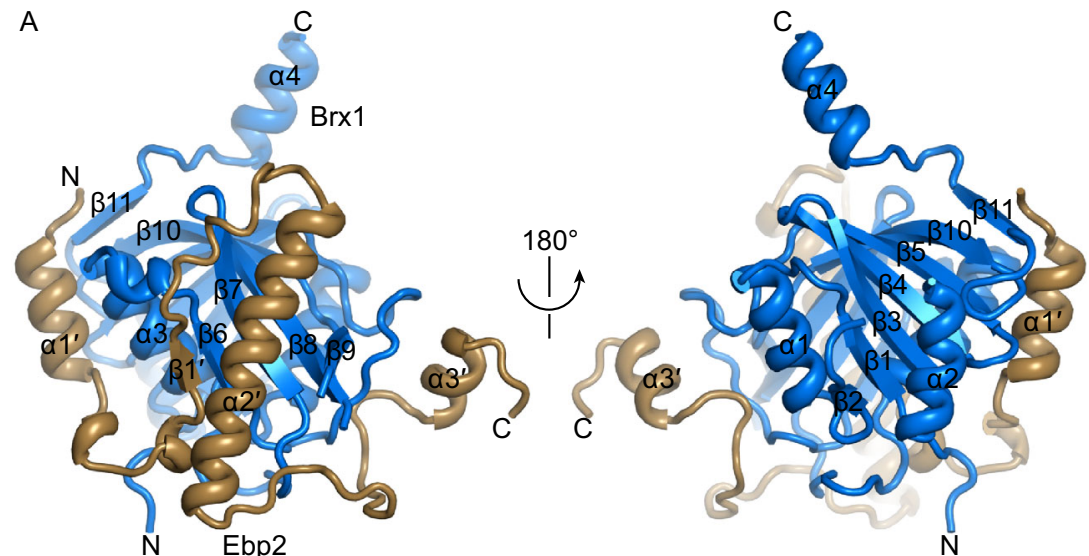

B

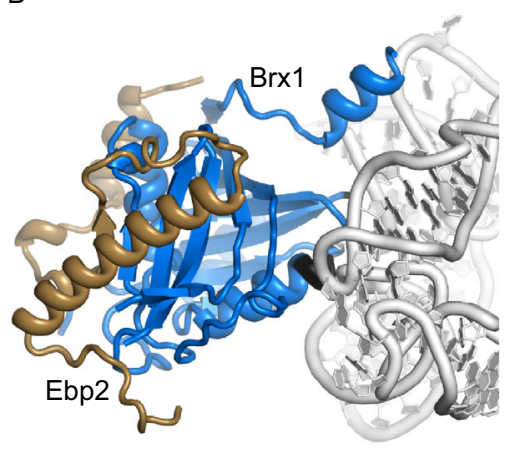

C

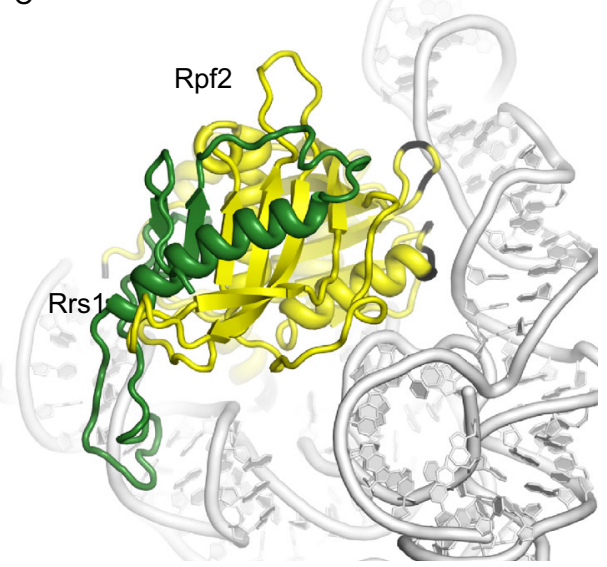

E

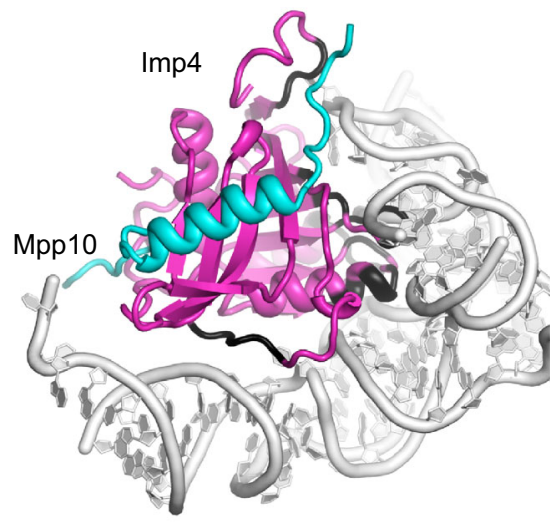

Many AFs and RPLs have been classified according to their role in pre-rRNA processing (Sahasranaman et al., 2011; Talkish et al., 2012; Woolford and Baserga, 2013; Gamalinda et al., 2014; de la Cruz et al., 2015). A3-factors (or early factors) are required for processing of $27 \mathrm{SA} 3$ to 27SB pre-rRNA, B-factors (or middle factors) are required for C2 cleavage of ITS2 and late factors are required for processing of $7 S$ pre-rRNA. Base on functional classification, binding dependence and structural location of RPLs, a hierarchical model has been proposed for 60S assembly with assembly beginning at the solvent side (Gamalinda et al., 2014). The Rpf1-TAP pre-60S structure is half- assembled at the solvent side and contains 8 of 13 AFs and all of 12 RPLs that are classified as early A3-factors (Figs. 2C and S1). This suggests that the structure present in this study corresponds to the predicted early assembly intermediate and is required for ITS1 processing.

By analyzing the association of AFs and RPLs with plasmid-derived pre-rRNA fragments of increasing length, the earliest precursor of 60S was found to form in a stepwise manner (Chen et al., 2017). The D2 factors Rpf1, Mak16, Nsa1 and Rrp1, which are initially recruited by a pre-rRNA fragment ending at domain II, are found to bind at the junction of domains I and II. This suggests that domains I and II 
A

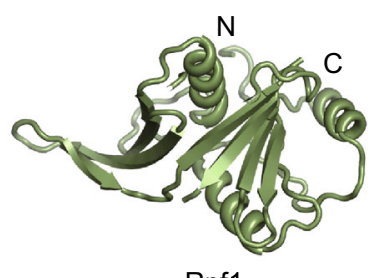

Rpf1
B

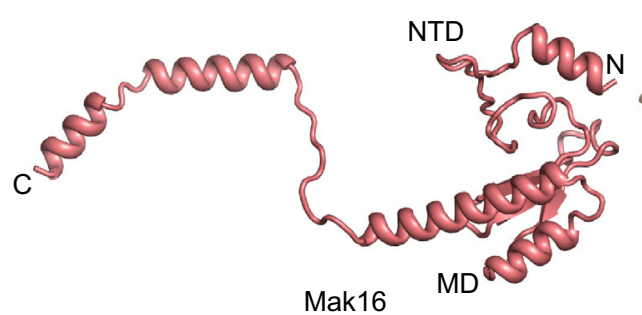

C

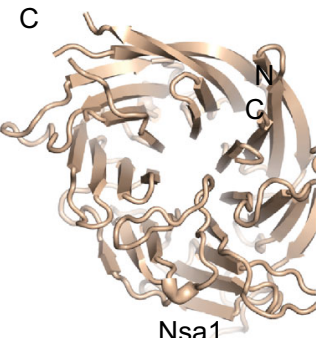

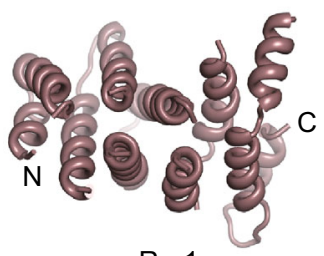

Rrp1

E

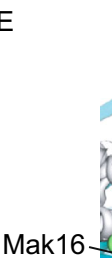

NTD

D2 L18

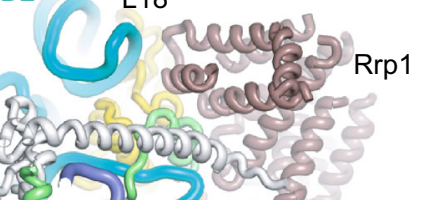

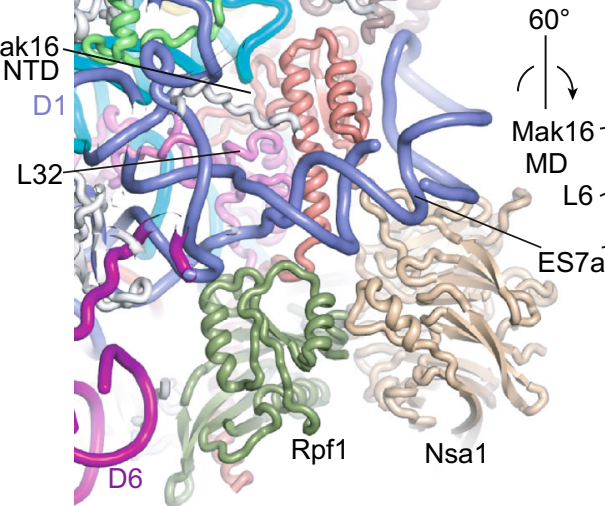

F

G

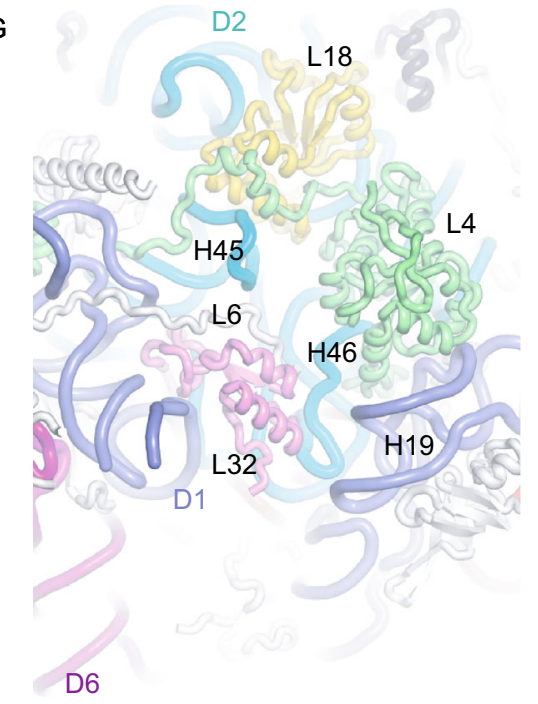

D2

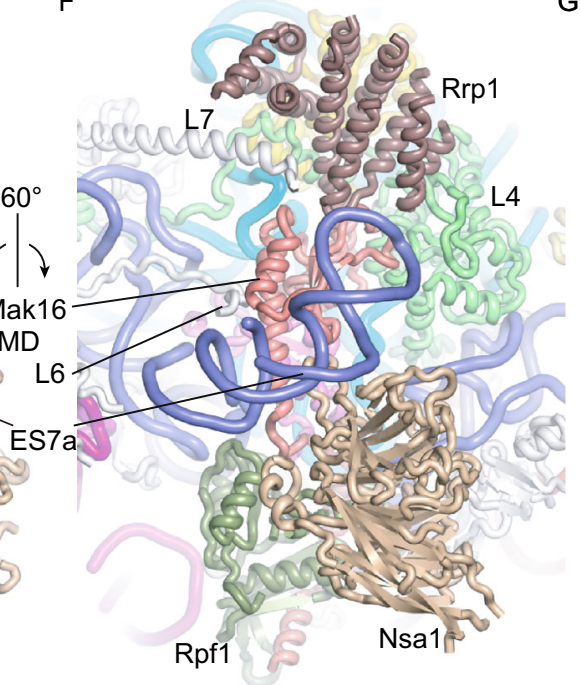

Figure 5. Assembly of D2 factors. (A-D) Structures of Rpf1 (A), Mak16 (B), Nsa1 (C) and Rrp1 (D). The N- and C-termini are labeled for each protein. The N-terminal domain (NTD) and middle domain (MD) of Mak16 are indicated. (E and F) Two views of Rpf1, Mak16, Nsa1 and Rrp1 assembled at the junction of domains I and II. AFs and individual domains of 25S rRNAs are color coded. L4, L18 and L32 are colored green, violet and yellow, respectively, and other RPLs are shown in gray. (G) Structure of mature 60S subunit (PDB code: 4V88) in the same view as (F).

could already associate despite the absence of other domains. This suggests a sequential assembly model for the Rpf1-TAP pre-60S. Domains I and II first combine into a structural core that is late joined by domain VI.

\section{Note added}

While this work was prepared for publication, the cryo-EM structures of nucleolar pre-60S in multiple states were reported (Kater et al., 2017). Our structure is mostly similar with state B derived from Nsa1 and Ytm1 affinity purification.

\section{MATERIALS AND METHODS}

Purification of Rpf1-TAP particles

The Rpf1-TAP strain (BY4741, RPF1-TAP::HisMX3) was purchased from Open Biosystems. Six liters of cells were grown at $30^{\circ} \mathrm{C}$ in YPD medium ( $1 \%$ yeast extract, $2 \%$ peptone, $2 \%$ glucose) to $\mathrm{OD}_{600}$ of 1-2. The cells were harvested and re-suspended in lysis buffer (20 $\mathrm{mmol} / \mathrm{L}$ HEPES-KOH, $\mathrm{pH} 8.0,110 \mathrm{mmol} / \mathrm{L} \mathrm{KOAc,} 40 \mathrm{mmol} / \mathrm{L} \mathrm{NaCl}$,
$0.1 \% \mathrm{NP}-40$ ) supplemented with one tablet of EDTA-free protease inhibitor cocktail (Roche). The cells were lysed by a high pressure cell disruptor $(\mathrm{JNBIO})$ and clarified by centrifugation at $6,000 \times \mathrm{g}$ for $10 \mathrm{~min}$. The supernatant was filtered through a $0.45 \mu \mathrm{m}$ membrane and incubated with $50 \mathrm{mg}$ of IgG-coated magnetic Dynabeads (Invitrogen) for $30 \mathrm{~min}$. The beads were washed with lysis buffer twice and incubated with TEV protease in cleavage buffer $(10 \mathrm{mmol} / \mathrm{L}$ Tris$\mathrm{HCl}, \mathrm{pH} 8.0,100 \mathrm{mmol} / \mathrm{L} \mathrm{NaCl}$ and $2 \mathrm{mmol} / \mathrm{L}$ DTT) at $4{ }^{\circ} \mathrm{C}$ for $4-8 \mathrm{~h}$. The released complex was concentrated to $\sim 50 \mu \mathrm{L}$.

\section{Cryo-EM data collection and image processing}

To prepare vitrified specimen, $3 \mu \mathrm{L}$ of sample $\left(\mathrm{OD}_{260}=3-5\right)$ was applied to glow-charged holey carbon grids (GiG R422) at $4{ }^{\circ} \mathrm{C}$ and $100 \%$ humidity using an FEI Vitrobot Mark IV. The grids were blotted for 1-3 s and rapidly plunged into liquid ethane. The cryogenic sample was first screened on a Talos F200C 200kV electron microscope equipped with a Ceta camera (FEI). A dataset was collected with a pixel size of $2.27 \AA$. Images were processed with RELION (Scheres, 2012a, b). Particles were picked, cleaned and subjected to $3 \mathrm{D}$ classification using the mature yeast $40 \mathrm{~S}$ ribosome 
as the initial model (Ben-Shem et al., 2011). A density map with clear structural feature was obtained at $11.7 \AA$ resolution. This map was used as the initial model for subsequent image processing.

High-resolution images were recorded on a Titan Krios (FEI) operated at $300 \mathrm{kV}$ and equipped with a Falcon III detector. A total of 1983 images were collected with SerialEM (Mastronarde, 2005) in low-dose mode at a pixel size of $1.38 \AA$. Images were recorded in 23 movie frames with an exposure time of $1.5 \mathrm{~s}$ and a total dose of $30 \mathrm{e} / \AA^{2}$ and corrected for motion with MOTIONCORR (Li et al., 2013). Contrast transfer function (CTF) values were measured with CTFFIND4 (Rohou and Grigorieff, 2015). A total of 1,786 goodquality images with a defocus range of 1-4 $\mu \mathrm{m}$ were chosen for particle picking in RELION. Particles were extracted with a box size of 360 pixels and downsized 4-fold for 3D classification. Three rounds of 3D classification were performed with a mask of $400 \AA$ diameter (Fig. S2). The classes with high resolution and similar features were combined for further classification or refinement. The final 3D refinement was conducted on 98,155 particles with the goldstandard Fourier shell correlation (FSC) method (Scheres and Chen, 2012). A density map at $3.65 \AA$ resolution was obtained after postprocessing, correction of modulation transfer function of the detector and B-factor sharping. FSC curves were calculated from data halfsets using soft masks and corrected for the effect of the mask using high-resolution noise substitution. The reported resolution was based on the gold-standard FSC $=0.143$ criterion. Local resolution was estimated with ResMap (Kucukelbir et al., 2014). To calculate FSC between model and map, the model was converted into a density map in Chimera, which was resampled to the grid of the map used for refinement. FSC between two maps was calculated with relion_image_handler. For cross-validation, the model was re-refined against a map reconstruction from half the data (Amunts et al., 2014).

\section{Model building}

The cryo-EM structure of Nog2-TAP pre-60S (PDB code 3JCT) was fit into the density as the starting model (Wu et al., 2016). The RNA and protein components without density were removed. The unmodeled density was assigned with assistance of the BALBESMOLREP pipeline (Brown et al., 2015) and CXMS data. The crystal structures of the Brx1 and Ebp2 complex determined here and the Nsa1 structure (Lo et al., 2017) were fitted as rigid body. Homology models of Rpf1 and Has 1 were created by SWISS-MODEL (Biasini et al., 2014). Rrp1 and Mak16 were manually built. A few unassigned densities were modeled as poly-alanine chains, and additional weak densities were not modeled. Nucleotides 438-493 of helix ES7a were modeled based on the structural prediction of RNAComposer (Popenda et al., 2012). The structure was further adjusted in COOT (Emsley and Cowtan, 2004) and refined in real space with secondary structure and geometry restraints using PHENIX (Adams et al., 2010). Structural figures were prepared with PyMOL 1.7 (Schrödinger, LLC) and Chimera (Pettersen et al., 2004).

\section{Expression and purification of the Brx1 and Ebp2 complex}

The genes of Brx1 (total 291 residues) and Ebp2 (total 427 residues) were amplified from yeast genomic DNA and cloned with the transfer-PCR approach (Erijman et al., 2011). Ebp2 and its fragments were cloned into a modified pET28a vector (Novagen) and fused to an N-terminal $\mathrm{His}_{6}-\mathrm{Smt} 3$ tag. $\mathrm{Brx} 1$ and its fragments were cloned into a modified pETDuet-1 vector and fused to an $\mathrm{N}$-terminal $\mathrm{His}_{6}$-GST tag followed by a PreScission cleavage site. The constructed plasmids were verified by DNA sequencing. Native or selenomethionine (SeMet)-labeled Brx1 and Ebp2 were co-expressed in the E. coli Rosetta 2(DE3) strain and co-purified with HisTrap, heparin and gel filtration chromatography according to the previously described procedure (Zheng and Ye, 2014). The interaction between the two proteins was confirmed by GST pull-down assay.

Crystallization and structural determination of the Brx1 and Ebp2 complex

Several fragments of Ebp2 and Brx1 were tested for crystallization. High quality crystals were grown from a complex containing residues 186-295 of Ebp2 and residues 26-259 of Ebp2. Both the native and SeMet-labeled complexes $(15 \mathrm{mg} / \mathrm{mL}$ in $10 \mathrm{mmol} / \mathrm{L}$ Tris- $\mathrm{HCl}, \mathrm{pH} 8.0$ and $500 \mathrm{mmol} / \mathrm{L} \mathrm{NaCl}$ ) were crystallized in $0.1 \mathrm{~mol} / \mathrm{L}$ Bis-Tris, $\mathrm{pH} 5.5$ and $1.8 \mathrm{~mol} / \mathrm{L}$ ammonium sulfate using the vapor diffusion hanging drop method. The crystals were cryo-protected in $20 \%$ glycerol made in the reservoir solution and flash frozen in liquid nitrogen.

Diffraction data were collected at the beamLine BL17U of Shanghai Synchrotron Radiation Facility and processed with HKL2000 (Otwinowski and Minor, 1997). Two types of crystals that belonged to $\mathrm{C} 2{ }_{1}$ or $\mathrm{P} 2_{1}$ space group were obtained at the same condition. The structure was first solved by the single-wavelength anomalous diffraction (SAD) method based on a $\mathrm{C} 2{ }_{1}$ Se-derivative dataset at $2.8 \AA$ resolution using SHARP (Vonrhein et al., 2007) (Table S3). The resultant electron density map was of moderate quality. The experimental phases were iteratively combined with the phases calculated from a partially built model to improve the map. A better dataset at $2.3 \AA$ resolution was later collected on the native $\mathrm{P} 2{ }_{1}$ crystal and used for final refinement. The model was built in COOT (Emsley and Cowtan, 2004) and refined with PHENIX (Adams et al., 2010). The final model contains two copies of the complex composed of Ebp2 residues 195-293, Brx1 residues 26$65,70-195$ and 210-255, 308 water molecules and one sulfate ion.

\section{Mass spectrometric analysis}

Mass spectrometric analysis was conducted as previously described (Zhang et al., 2016). The total spectral counts per 100 residues (SCPHR) were calculated for each identified protein and further normalized against Brx1, Ebp2, Erb1, Ytm1, Nop7, Cic1 and Has1, yielding the relative spectral abundance factor (RSAF) (Supplementary Dataset 1).

\section{CXMS}

CXMS was performed as described (Sun et al., 2017). The Rpf1TAP sample containing $\sim 10 \mu \mathrm{g}$ of total proteins was crosslinked with $\mathrm{BS}^{3}$ or DSS, precipitated with acetone and digested by trypsin. The sample was analyzed by LC-MS/MS on an EASY-nLC 1000 system interfaced to a Q-Exactive HF mass spectrometer (Thermo Fisher Scientific). Cross-linked peptides were identified with pLink (Yang et al., 2012). 
Protein interaction analysis of Rpf1 and Mak16

Yeast two-hybrid and GST pull-down assays were conducted as described (Zheng et al., 2014). Rpf1 was cloned into a modified pET28a vector (Novagen) and fused to an $\mathrm{N}$-terminal $\mathrm{His}_{6}-\mathrm{Smt} 3$ tag. Mak16 and its fragments were cloned into a modified pETDuet-1 vector and fused to an N-terminal $\mathrm{His}_{6}$-GST tag followed by a PreScission cleavage site. For GST pull-down assay, Rpf1 was expressed and purified by HisTrap chromatography, Ulp1 cleavage of the $\mathrm{His}_{6}-\mathrm{Smt} 3 \mathrm{tag}$, heparin and gel filtration chromatography. Mak16 was purified by HisTrap chromatography without cleavage of the tag. To purify the complex, Rpf1 and Mak16 were co-expressed and co-purified according to the previously described procedure (Zheng and Ye, 2014).

\section{Accession numbers}

The cryo-EM density map, coordinates and structural factors have been deposited to EMDB and PDB under accession numbers EMD$6878,5 Z 3 G$ and $5 Z 1 G$.

\section{ACKNOWLEDGEMENTS}

We thank the Center for Biological Imaging (CBI), Institute of Biophysics, Chinese Academy of Sciences for cryo-EM study and HPCService Station in CBI for image processing. We are grateful to Fei Sun, Xiaojun Huang, Zhenxi Guo, Gang Ji, Bolin Zhu, Shuoguo Li and Deyin Fan at CBI for help in EM sample preparation and data collection, Gaihong Cai and She Chen at the Proteomics Center of NIBS for mass spectrometry analysis and the staffs at beamLine BL17U of National Facility for Protein Sciences Shanghai and Shanghai Synchrotron Radiation Facility for assistance in data collection. The study was supported by Strategic Priority Research Program of Chinese Academy of Sciences (XDB08010203), National Key R\&D Program of China (2017YFA0504600), the National Natural Science Foundation of China (Grant Nos. 31430024, 91540201 and 31325007), the Ministry of Science and Technology of China (973 grant 2014CB84980001 to Meng-Qiu Dong) and 100 Talents Program of CAS.

\section{ABBREVIATIONS}

$\mathrm{AF}$, assembly factor; CXMS, chemical crosslinking and mass spectrometry; $\mathrm{CP}$, central protuberance; cryo-EM, cryo-electron microscopy; ES, extension segment; ETS, external transcribed spacer; ITS, internal transcribed spacer; LSU, large subunit; prerRNA, precursor ribosomal RNA; RPL, large subunit ribosomal protein; RSAF, relative spectral abundance factor; SCPHR, spectral counts per 100 residues; SSU, small subunit; TAP, tandem affinity purification.

\section{COMPLIANCE WITH ETHICS GUIDELINES}

Dejian Zhou, Xing Zhu, Sanduo Zheng, Dan Tan, Meng-Qiu Dong and Keqiong Ye declare no competing financial interests. This article does not contain any studies with human or animal subjects performed by any of the authors.

\section{OPEN ACCESS}

This article is distributed under the terms of the Creative Commons Attribution 4.0 International License (http://creativecommons.org/ licenses/by/4.0/), which permits unrestricted use, distribution, and reproduction in any medium, provided you give appropriate credit to the original author(s) and the source, provide a link to the Creative Commons license, and indicate if changes were made.

\section{REFERENCES}

Adams PD, Afonine PV, Bunkoczi G, Chen VB, Davis IW, Echols N, Headd JJ, Hung LW, Kapral GJ, Grosse-Kunstleve RW et al (2010) PHENIX: a comprehensive Python-based system for macromolecular structure solution. Acta Crystallogr D Biol Crystallogr 66:213-221

Amunts A, Brown A, Bai XC, Llacer JL, Hussain T, Emsley P, Long F, Murshudov G, Scheres SHW, Ramakrishnan V (2014) Structure of the yeast mitochondrial large ribosomal subunit. Science 343:1485-1489

Asano N, Kato K, Nakamura A, Komoda K, Tanaka I, Yao M (2015) Structural and functional analysis of the Rpf2-Rrs1 complex in ribosome biogenesis. Nucleic Acids Res 43:4746-4757

Barandun J, Chaker-Margot M, Hunziker M, Molloy KR, Chait BT, Klinge $S$ (2017) The complete structure of the small-subunit processome. Nat Struct Mol Biol 24:944-953

Barrio-Garcia C, Thoms M, Flemming D, Kater L, Berninghausen O, Bassler J, Beckmann R, Hurt E (2016) Architecture of the Rix1Rea1 checkpoint machinery during pre-60S-ribosome remodeling. Nat Struct Mol Biol 23:37-44

Bassler J, Kallas M, Pertschy B, Ulbrich C, Thoms M, Hurt E (2010) The AAA-ATPase Rea1 drives removal of biogenesis factors during multiple stages of $60 \mathrm{~S}$ ribosome assembly. Mol Cell 38:712-721

Bassler J, Ahmed YL, Kallas M, Kornprobst M, Calvino FR, Gnadig M, Thoms M, Stier G, Ismail S, Kharde S et al (2017) Interaction network of the ribosome assembly machinery from a eukaryotic thermophile. Protein Sci 26:327-342

Ben-Shem A, Garreau de Loubresse N, Melnikov S, Jenner L, Yusupova G, Yusupov M (2011) The structure of the eukaryotic ribosome at 3.0 A resolution. Science 334:1524-1529

Biasini M, Bienert S, Waterhouse A, Arnold K, Studer G, Schmidt T, Kiefer F, Gallo Cassarino T, Bertoni M, Bordoli L et al (2014) SWISS-MODEL: modelling protein tertiary and quaternary structure using evolutionary information. Nucleic Acids Res 42:W252258

Bradatsch B, Leidig C, Granneman S, Gnadig M, Tollervey D, Bottcher B, Beckmann R, Hurt E (2012) Structure of the pre-60S ribosomal subunit with nuclear export factor Arx1 bound at the exit tunnel. Nat Struct Mol Biol 19:1234-1241

Brown A, Long F, Nicholls RA, Toots J, Emsley P, Murshudov G (2015) Tools for macromolecular model building and refinement into electron cryo-microscopy reconstructions. Acta Crystallogr D Biol Crystallogr 71:136-153

Chaker-Margot M, Hunziker M, Barandun J, Dill BD, Klinge S (2015) Stage-specific assembly events of the 6-MDa small-subunit 
processome initiate eukaryotic ribosome biogenesis. Nat Struct Mol Biol 22:920-923

Chaker-Margot, M., Barandun, J., Hunziker, M., and Klinge, S. (2017). Architecture of the yeast small subunit processome. Science 355 , eaal 1880 .

Chen W, Xie Z, Yang F, Ye K (2017) Stepwise assembly of the earliest precursors of large ribosomal subunits in yeast. Nucleic Acids Res 45:6837-6847

Cheng J, Kellner N, Berninghausen O, Hurt E, Beckmann R (2017) 3.2-A-resolution structure of the $90 \mathrm{~S}$ preribosome before $\mathrm{A} 1$ prerRNA cleavage. Nat Struct Mol Biol 24:954-964

de la Cruz J, Karbstein K, Woolford JL Jr (2015) Functions of ribosomal proteins in assembly of eukaryotic ribosomes in vivo. Annu Rev Biochem 84:93-129

Del Campo M, Lambowitz AM (2009) Structure of the Yeast DEAD box protein Mss116p reveals two wedges that crimp RNA. Mol Cell 35:598-609

Dembowski JA, Kuo B, Woolford JL Jr (2013) Has1 regulates consecutive maturation and processing steps for assembly of 60S ribosomal subunits. Nucleic Acids Res 41:7889-7904

Dragon F, Gallagher JE, Compagnone-Post PA, Mitchell BM, Porwancher KA, Wehner KA, Wormsley S, Settlage RE, Shabanowitz J, Osheim $Y$ et al (2002) A large nucleolar U3 ribonucleoprotein required for $18 \mathrm{~S}$ ribosomal RNA biogenesis. Nature 417:967-970

Emery B, de la Cruz J, Rocak S, Deloche O, Linder P (2004) Has1p, a member of the DEAD-box family, is required for $40 \mathrm{~S}$ ribosomal subunit biogenesis in Saccharomyces cerevisiae. Mol Microbiol 52:141-158

Emsley P, Cowtan K (2004) Coot: model-building tools for molecular graphics. Acta Crystallogr D Biol Crystallogr 60:2126-2132

Erijman A, Dantes A, Bernheim R, Shifman JM, Peleg Y (2011) Transfer-PCR (TPCR): a highway for DNA cloning and protein engineering. J Struct Biol 175(2):171-177

Falk S, Tants JN, Basquin J, Thoms M, Hurt E, Sattler M, Conti E (2017) Structural insights into the interaction of the nuclear exosome helicase Mtr4 with the preribosomal protein Nop53. RNA 23:1780-1787

Fatica A, Cronshaw AD, Dlakic M, Tollervey D (2002) Ssf1p prevents premature processing of an early pre-60S ribosomal particle. Mol Cell 9:341-351

Gamalinda M, Ohmayer U, Jakovljevic J, Kumcuoglu B, Woolford J, Mbom B, Lin L, Woolford JL Jr (2014) A hierarchical model for assembly of eukaryotic $60 \mathrm{~S}$ ribosomal subunit domains. Genes Dev 28:198-210

Grandi P, Rybin V, Bassler J, Petfalski E, Strauss D, Marzioch M, Schafer T, Kuster B, Tschochner H, Tollervey D et al (2002) $90 \mathrm{~S}$ pre-ribosomes include the $35 \mathrm{~S}$ pre-rRNA, the U3 snoRNP, and 40S subunit processing factors but predominantly lack $60 \mathrm{~S}$ synthesis factors. Mol Cell 10:105-115

Greber BJ, Gerhardy S, Leitner A, Leibundgut M, Salem M, Boehringer D, Leulliot N, Aebersold R, Panse VG, Ban N (2015) Insertion of the biogenesis factor Rei1 probes the ribosomal tunnel during 60S maturation. Cell 164:91-102

Kater L, Thoms M, Barrio-Garcia C, Cheng J, Ismail S, Ahmed YL, Bange G, Kressler D, Berninghausen O, Sinning I et al (2017)
Visualizing the assembly pathway of nucleolar Pre-60S ribosomes. Cell 171(1599-1610):e1514

Kharde S, Calvino FR, Gumiero A, Wild K, Sinning I (2015) The structure of Rpf2-Rrs1 explains its role in ribosome biogenesis. Nucleic Acids Res 43:7083-7095

Klinge S, Voigts-Hoffmann F, Leibundgut M, Arpagaus S, Ban N (2011) Crystal structure of the eukaryotic 60S ribosomal subunit in complex with initiation factor 6. Science 334:941-948

Kornprobst M, Turk M, Kellner N, Cheng J, Flemming D, Kos-Braun I, Kos M, Thoms M, Berninghausen O, Beckmann R et al (2016) Architecture of the $90 \mathrm{~S}$ pre-ribosome: a structural view on the birth of the eukaryotic ribosome. Cell 166:380-393

Kressler D, Roser D, Pertschy B, Hurt E (2008) The AAA ATPase Rix7 powers progression of ribosome biogenesis by stripping Nsa1 from pre-60S particles. J Cell Biol 181:935-944

Kucukelbir A, Sigworth FJ, Tagare HD (2014) Quantifying the local resolution of cryo-EM density maps. Nat Methods 11:63-65

Leidig C, Thoms M, Holdermann I, Bradatsch B, Berninghausen O, Bange G, Sinning I, Hurt E, Beckmann R (2014) 60S ribosome biogenesis requires rotation of the $5 S$ ribonucleoprotein particle. Nat Commun 5:3491

Li X, Mooney P, Zheng S, Booth CR, Braunfeld MB, Gubbens S, Agard DA, Cheng $Y$ (2013) Electron counting and beam-induced motion correction enable near-atomic-resolution single-particle cryo-EM. Nat Methods 10:584-590

Lo YH, Romes EM, Pillon MC, Sobhany M, Stanley RE (2017) Structural analysis reveals features of ribosome assembly factor Nsa1/WDR74 important for localization and interaction with Rix7/ NVL2. Structure 25:762-772

Ma C, Wu S, Li N, Chen Y, Yan K, Li Z, Zheng L, Lei J, Woolford JL Jr, Gao N (2017) Structural snapshot of cytoplasmic pre-60S ribosomal particles bound by Nmd3, Lsg1, Tif6 and Reh1. Nat Struct Mol Biol 24:214-220

Madru C, Lebaron S, Blaud M, Delbos L, Pipoli J, Pasmant E, Rety S, Leulliot N (2015) Chaperoning 5S RNA assembly. Genes Dev 29:1432-1446

Mallam AL, Del Campo M, Gilman B, Sidote DJ, Lambowitz AM (2012) Structural basis for RNA-duplex recognition and unwinding by the DEAD-box helicase Mss116p. Nature 490:121-125

Malyutin AG, Musalgaonkar S, Patchett S, Frank J, Johnson AW (2017) Nmd3 is a structural mimic of elF5A, and activates the cpGTPase Lsg1 during $60 \mathrm{~S}$ ribosome biogenesis. EMBO J 36:854-868

Mastronarde DN (2005) Automated electron microscope tomography using robust prediction of specimen movements. J Struct Biol 152:36-51

Matsuo Y, Granneman S, Thoms M, Manikas RG, Tollervey D, Hurt E (2014) Coupled GTPase and remodelling ATPase activities form a checkpoint for ribosome export. Nature 505:112-116

McCann KL, Charette JM, Vincent NG, Baserga SJ (2015) A protein interaction map of the LSU processome. Genes Dev 29:862-875

$\mathrm{Ng} \mathrm{CL}$, Waterman D, Koonin EV, Antson AA, Ortiz-Lombardia M (2005) Crystal structure of Mil (Mth680): internal duplication and similarity between the Imp4/Brix domain and the anticodonbinding domain of class Ila aminoacyl-tRNA synthetases. EMBO Rep 6:140-146 
Osheim YN, French SL, Keck KM, Champion EA, Spasov K, Dragon F, Baserga SJ, Beyer AL (2004) Pre-18S ribosomal RNA is structurally compacted into the SSU processome prior to being cleaved from nascent transcripts in Saccharomyces cerevisiae. Mol Cell 16:943-954

Otwinowski Z, Minor W (1997) Processing of X-ray diffraction data collected in oscillation mode. Methods Enzymol 276:307-326

Pena C, Hurt E, Panse VG (2017) Eukaryotic ribosome assembly, transport and quality control. Nat Struct Mol Biol 24:689-699

Pettersen EF, Goddard TD, Huang CC, Couch GS, Greenblatt DM, Meng EC, Ferrin TE (2004) UCSF Chimera-a visualization system for exploratory research and analysis. J Comput Chem 25:1605-1612

Popenda M, Szachniuk M, Antczak M, Purzycka KJ, Lukasiak P, Bartol N, Blazewicz J, Adamiak RW (2012) Automated 3D structure composition for large RNAs. Nucleic Acids Res 40:e112

Rohou A, Grigorieff N (2015) CTFFIND4: fast and accurate defocus estimation from electron micrographs. J Struct Biol 192:216-221

Sahasranaman A, Dembowski J, Strahler J, Andrews P, Maddock J, Woolford JL Jr (2011) Assembly of Saccharomyces cerevisiae $60 S$ ribosomal subunits: role of factors required for $27 \mathrm{~S}$ pre-rRNA processing. EMBO J 30:4020-4032

Sa-Moura B, Kornprobst M, Kharde S, Ahmed YL, Stier G, Kunze R, Sinning I, Hurt E (2017) Mpp10 represents a platform for the interaction of multiple factors within the $90 \mathrm{~S}$ pre-ribosome. PLoS ONE 12:e0183272

Scheres SH (2012a) A Bayesian view on cryo-EM structure determination. J Mol Biol 415:406-418

Scheres SH (2012b) RELION: implementation of a Bayesian approach to cryo-EM structure determination. J Struct Biol 180:519-530

Scheres SH, Chen S (2012) Prevention of overfitting in cryo-EM structure determination. Nat Methods 9:853-854

Shimoji K, Jakovljevic J, Tsuchihashi K, Umeki Y, Wan K, Kawasaki S, Talkish J, Woolford JL Jr, Mizuta K (2012) Ebp2 and Brx1 function cooperatively in $60 \mathrm{~S}$ ribosomal subunit assembly in Saccharomyces cerevisiae. Nucleic Acids Res 40:4574-4588

Sun Q, Zhu X, Qi J, An W, Lan P, Tan D, Chen R, Wang B, Zheng S, Zhang $C$ et al (2017) Molecular architecture of the $90 \mathrm{~S}$ small subunit pre-ribosome. Elife 6:e22086

Talkish J, Zhang J, Jakovljevic J, Horsey EW, Woolford JL Jr (2012) Hierarchical recruitment into nascent ribosomes of assembly factors required for 27SB pre-rRNA processing in Saccharomyces cerevisiae. Nucleic Acids Res 40:8646-8661

Thoms M, Thomson E, Bassler J, Gnadig M, Griesel S, Hurt E (2015) The exosome is recruited to RNA substrates through specific adaptor proteins. Cell 162:1029-1038

Ulbrich C, Diepholz M, Bassler J, Kressler D, Pertschy B, Galani K, Bottcher B, Hurt E (2009) Mechanochemical removal of ribosome biogenesis factors from nascent $60 \mathrm{~S}$ ribosomal subunits. Cell 138:911-922

Vonrhein C, Blanc E, Roversi P, Bricogne G (2007) Automated structure solution with autoSHARP. Methods Mol Biol 364:215230

Wehner KA, Baserga SJ (2002) The sigma(70)-like motif: a eukaryotic RNA binding domain unique to a superfamily of proteins required for ribosome biogenesis. Mol Cell 9:329-339

Weis F, Giudice E, Churcher M, Jin L, Hilcenko C, Wong CC, Traynor D, Kay RR, Warren AJ (2015) Mechanism of elF6 release from the nascent $60 \mathrm{~S}$ ribosomal subunit. Nat Struct Mol Biol 22:914-919

Woolford JL Jr, Baserga SJ (2013) Ribosome biogenesis in the yeast Saccharomyces cerevisiae. Genetics 195:643-681

Wu S, Tutuncuoglu B, Yan K, Brown H, Zhang Y, Tan D, Gamalinda M, Yuan Y, Li Z, Jakovljevic J et al (2016) Diverse roles of assembly factors revealed by structures of late nuclear pre-60S ribosomes. Nature 534:133-137

Yang Q, Del Campo M, Lambowitz AM, Jankowsky E (2007) DEADbox proteins unwind duplexes by local strand separation. Mol Cell 28:253-263

Yang B, Wu YJ, Zhu M, Fan SB, Lin J, Zhang K, Li S, Chi H, Li YX, Chen HF, Luo SK, Ding YH, Wang LH, Hao Z, Xiu LY, Chen S, Ye K, He SM, Dong MQ (2012) Identification of cross-linked peptides from complex samples. Nat Methods 9(9):904-906

Zhang L, Wu C, Cai G, Chen S, Ye K (2016) Stepwise and dynamic assembly of the earliest precursors of small ribosomal subunits in yeast. Genes Dev 30:718-732

Zheng S, Ye K (2014) Purification, crystallization and preliminary $X$-ray diffraction analysis of Imp3 in complex with an Mpp10 peptide involved in yeast ribosome biogenesis. Acta Crystallogr $F$ Struct Biol Commun 70:918-921

Zheng S, Lan P, Liu X, Ye K (2014) Interaction between ribosome assembly factors Krr1 and Faf1 is essential for formation of small ribosomal subunit in yeast. J Biol Chem 289:22692-22703 